\title{
BÚSQUEDA DE CAPACIDAD PRODUCTORA DE BIOSURFACTANTES EN ACTINOBACTERIAS HALOALCALÓFILAS Y HALOALCALOTOLERANTES
}

\author{
Reyna del Carmen LARA-SEVERINO ${ }^{1}$, Leobardo Manuel GÓMEZ OLIVAN², \\ Ángel Horacio SANDOVAL TRUJILLO ${ }^{3}$, Keila ISAAC-OLIVE ${ }^{1}$ y Ninfa RAMÍREZ-DURÁN ${ }^{1 *}$
}

${ }^{1}$ Facultad de Medicina, Universidad Autónoma del Estado de México. Paseo Tollocan esquina Jesús Carranza, Colonia Moderna de la Cruz, Toluca, México, C. P. 50180

${ }^{2}$ Facultad de Química, Universidad Autónoma del Estado de México. Paseo Colón, Colonia Residencial Colón, Toluca, México, C. P. 50120

${ }^{3}$ Departamento de Sistemas Biológicos, Universidad Autónoma Metropolitana, Campus Xochimilco. Calzada del Hueso 1100, Colonia Villa Quietud, Delegación Coyoacán, Ciudad de México, México, C. P. 04960

*Autor para correspondencia: ninfard@hotmail.com

(Recibido noviembre 2015; aceptado diciembre 2016)

Palabras clave: bacteria, emulsificante, tensoactivo, contaminación, biorremediación

\section{RESUMEN}

Una opción para biorremediar ambientes salinos contaminados con compuestos orgánicos hidrofóbicos, es el uso de microorganismos haloalcalófilos o haloalcalotolerantes capaces de producir biosurfactantes estables y consumir al compuesto hidrófobo. El biosurfactante solubiliza al contaminante haciéndolo biodisponible para el microrganismo. De estudios previos se conocía la capacidad de siete actinobacterias haloalcalotolerantes para transformar el antraceno. En este trabajo, a estas siete más 11 más aisladas de ambientes salinos, se les determinó la capacidad para producir biosurfactantes con propiedades emulsificantes. A las que la mostraron, fueron seleccionadas para saber si utilizaban al antraceno como única fuente de carbono y energía. La capacidad para producir biosurfactante fue determinada por los métodos de actividad hemolítica, gota colapsada y difusión del diámetro de la gota. La propiedad emulsificante y la estabilidad de la emulsión se caracterizaron con el índice de emulsificación y el índice de emulsificación relativo, respectivamente. De 18 actinobacterias evaluadas, cinco cepas mostraron capacidad para producir biosurfactantes con propiedades emulsificantes, alta estabilidad en la emulsión y utilización de antraceno como única fuente de carbono y energía. De estas cinco, dos son haloalcalófilas pertenecientes a la especie Nocardiopsis salina y tres son haloalcalotolerantes pertenecientes a las especies: Kocuria palustris, Microbacterium testaceum y Kocuria rosea. En un estudio previo se determinó la capacidad transformadora de antraceno en las tres cepas haloalcalotolerantes, por lo tanto los resultados de este estudio sugieren proponerlas como candidatas para procesos de biorremediación de ambientes salinos contaminados con compuestos hidrófobos. Asimismo se sugiere determinar la misma capacidad para las dos cepas haloalcalófilas.

Key words: bacteria, emulsifier, surfactant, contamination, bioremediation 


\begin{abstract}
One option for bioremediation of saline environments contaminated with hydrophobic organic compounds is the use of haloalkaliphilic or haloalkalitolerant microorganisms capable of producing stable biosurfactants and consuming the hydrophobic compound. The biosurfactant solubilizes the contaminant making it bioavailable for the microorganism. Previous studies have revealed the ability to transform anthracene from seven haloalkalitolerant actinobacteria. In this work the ability to produce biosurfactants with emulsifying properties from these seven plus 11 isolates of saline environments were determined. Those that showed the capability were investigated for anthracene as their only source of carbon and energy. The ability to produce biosurfactant was determined by methods of hemolytic activity, collapsed droplet and diffusion of droplet diameter. The emulsifying property and the stability of the emulsion were characterized by the emulsification index and the relative emulsification index, respectively. Of 18 actinobacteria evaluated, five strains showed capacity to produce biosurfactants with emulsifying properties, high stability in the emulsion and the use of anthracene as their only source of carbon and energy. Of these five strains, two are haloalkaliphilic, they belong to the species Nocardiopsis salina and three are haloalkalitolerant, they belong to the species Kocuria palustris, Microbacterium testaceum and Kocuria rosea. In a previous study the transforming capacity of anthracene of the three haloalkalitolerant strains was determined; therefore the results of this study suggest they can be proposed as candidates for bioremediation processes of saline environments contaminated with hydrophobic compounds. Likewise it is also recommended to determine the same capacity for the two haloalkaliphilic strains.
\end{abstract}

\section{INTRODUCCIÓN}

La contaminación ambiental con petróleo y productos petroquímicos es reconocida como uno de los problemas actuales más importantes (Jain et al. 2011). El petróleo es una mezcla compleja de hidrocarburos y compuestos relacionados, clasificados como: alifáticos, asfaltenos e hidrocarburos policíclicos aromáticos (HPA) (Redman et al. 2012). Los HPA son un grupo de contaminantes orgánicos tóxicos que se han acumulado en el ambiente y muchos de ellos tienen propiedades mutagénicas y carcinogénicas (Juhasz y Naidu 2000, Zhong et al. 2007).

Una opción para limpiar el ambiente de HPA es a través del uso de surfactantes sintéticos o naturales. Estos compuestos son moléculas anfifilicas que tienen propiedades tensoactivas, emulsificantes y dispersantes. Reducen la tensión superficial y la tensión interfacial mediante la acumulación de fluidos inmiscibles en la interfase, lo que aumenta la solubilidad y movilidad de estos compuestos orgánicos hidrófobos e insolubles (Mulligan 2005, Zhu y Aitken 2010, Yañez-Ocampo y Wong-Villarreal 2013). Sin embargo, los surfactantes sintéticos a menudo son tóxicos, lo que representa una fuente adicional de contaminación (Bognolo 1999, Yañez-Ocampo y Wong-Villarreal 2013). Por esta razón se prefiere el uso de surfactantes de origen natural (biosurfactantes) ya que presentan poca o nula toxicidad, son altamente biodegradables (Soberón-Chávez y Maier 2011) y estables en condiciones extremas de temperaturas, pH y salinidad (Mukherjee et al. 2006).

Otra opción de eliminación de HPA es la biorremediación con microorganismos (Lai et al. 2009). Este proceso es más eficiente si los microrganismos tienen la capacidad de producir biosurfactantes, pues se mejora la biodisponibilidad y se facilita la asimilación de estos sustratos insolubles al incrementar su solubilidad (Batista et al. 2006).

Existen microorganismos capaces de desarrollarse en ambientes con condiciones extremas, como los que se caracterizan por su alta concentración de sal y valores altos de $\mathrm{pH}$. De acuerdo con las condiciones fisiológicas de salinidad y de $\mathrm{pH}$ que requieren los microorganismos para su crecimiento óptimo, éstos se clasifican como halófilos, halotolerantes, alcalófilos o alcalotolerantes. Los microorganismos halófilos son aquellos que requieren determinadas concentraciones de $\mathrm{NaCl}$ para crecer y vivir (Oren 2005). Los halotolerantes pueden crecer en medios con muy bajas concentraciones de $\mathrm{NaCl}$, incluso cero o bien tan altas como un $25 \%$ (Kushner y Kamekura 1988). Un microorganismo alcalófilo o alcalotolerante es el que requiere o tolera, respectivamente para su crecimiento óptimo un valor de $\mathrm{pH}$ de 9 o más alto (Horikoshi 1999). Uno haloalcalófilo es el que necesita tanto una 
concentración de $\mathrm{NaCl}$ determinada (hasta $30 \%$ ) y un $\mathrm{pH}$ alcalino ( $\mathrm{pH}$ 9) (Horikoshi 1999). Por otro lado, un microorganismo haloalcalotolerante es el que puede vivir ya sea en ausencia o en presencia de sal, incluso puede tolerar altas concentraciones de $\mathrm{NaCl}$ (hasta 25 \%) y también es capaz de crecer de manera óptima en un amplio rango de $\mathrm{pH}$ (8-9 o superior).

Kügler et al. (2015) recopiló las especies de actinobacterias productoras de biosurfactantes. Aunque la primera de ellas data de 1969, el número de especies identificadas al día de hoy con este fin no es numeroso. Con excepción del trabajo de Sarafin et al. (2014) los artículos publicados relacionados con este tema no especifican si los actinobacterias son halalcalófilas o haloalcalotolerantes. Estas bacterias además presentan capacidad para biodegradar contaminantes orgánicos (Ferradji et al. 2014). Algunas especies de actinobacterias productoras de biosurfactante son: Kocuria rosea (Mahjoubi et al. 2013), Nocardia otitidiscaviarum (Vyas y Dave 2011), Microbacterium sp. (Aniszewski et al. 2010), Nocardiopsis alba (Gandhimathi et al. 2009) y Nocardiopsis lucentensis (Kiran et al. 2010). Las especies Kocuria rosea, Microbacterium marinilacus, Nocardia pneumoniae, Nocardidopsis sp. y Nocardia otitidiscaviarum han sido identificadas como biodegradadoras de petróleo o HPA (Dixit y Pant 2000, Ahmed et al. 2010, Vyas y Dave 2011, Al-Awadhi et al. 2012), las cepas de Kocuria rosea y Nocardia otitidiscaviarum cumplen con ambas condiciones, son tanto biodegradadoras como productoras de biosurfactantes.

El antraceno es un HPA de tres anillos. La Agencia de Protección Ambiental de Estados Unidos (USEPA, por sus siglas en inglés) lo clasifica como un contaminante ambiental de prioridad. Sin embargo, no hay información suficiente para clasificarlo como carcinogénico (USEPA 1990). Por otro lado, la semejanza en su estructura a HPA carcinogénicos como benzo $[a]$ pireno y benzo $[a]$ antraceno es la principal razón para comúnmente utilizarlo como modelo de HPA en estudios de degradación (Bonnet et al. 2005).

Recientemente, a siete cepas de actinobacterias aisladas de suelos cercanos a áreas con actividad petrolera en el estado de Veracruz y que pertenecen a las especies Nocardia farcinica, Kocuria rosea, Kocuria palustris y Microbacterium testaceum, se les determinó por fluorescencia la capacidad de utilizar al antraceno como única fuente de carbono y energía y transformarlo (Lara-Severino et al. 2016a, 2016b). Sin embargo, su capacidad para producir biosurfactantes no ha sido estudiada.
El objetivo de este trabajo fue determinar la capacidad de cepas de actinobacterias aisladas en diferentes ambientes de México para producir biosurfactantes. Siete de estas cepas son las actinobacterias previamente identificadas y aisladas de Veracruz, cuya capacidad para transformar antraceno ya fue probada. Las cepas restantes provienen del suelo salino alcalino del ex lago de Texcoco, las cuales fueron identificadas genéticamente utilizando la secuenciación del gen 16S ARNr. Aquellas cepas que mostraron la facultad de producir biosurfactante con propiedades emulsificantes fueron seleccionadas para determinar además, su capacidad en utilizar al antraceno como única fuente de carbono y energía.

\section{MATERIALES Y MÉTODOS}

\section{Muestreo}

Se realizó un muestreo de tipo aleatorio, en suelos salino alcalinos del ex lago de Texcoco.

Las muestras fueron inoculadas en el medio de cultivo para microrganismos halófilos $(\mathrm{MH}): 10 \mathrm{~g} / \mathrm{L}$ de extracto de levadura, $5 \mathrm{~g} / \mathrm{L}$ de peptona proteosa, $1 \mathrm{~g} / \mathrm{L}$ de glucosa, $18 \mathrm{~g} / \mathrm{L}$ de agar, $10 \%$ de $\mathrm{NaCl}, \mathrm{pH}$ 7 (Quesada et al. 1983).

\section{Cepas}

Además de las cepas de actinobacterias aisladas de las muestras de suelo del ex lago de Texcoco, se utilizaron otras siete cepas de actinobacterias pertenecientes a las especies: Kocuria rosea, Kocuria palustris, Microbacterium testaceum y cuatro cepas de la especie Nocardia farcinica. Estas cepas fueron previamente aisladas de suelos cercanos a áreas con actividad petrolera en el estado de Veracruz y también fueron cultivadas en medio MH (Lara-Severino et al. 2016a).

\section{Aislamiento de las cepas del ex lago de Texcoco}

La selección de las cepas se realizó de acuerdo con las características morfológicas reportadas para actinobacterias en el manual de Bergey (Holt et al. 1994). Para confirmar la presencia de cepas de actinobacterias, se realizó la tinción de Gram con el fin de observar células Gram-positivas filamentosas y ramificadas, para lo cual se utilizó un microscopio Leica 5605.

Las cepas aisladas del ex lago de Texcoco y las siete cepas previamente identificadas y provenientes de suelos cercanos a aéreas con actividad petrolera del estado de Veracruz, fueron sometidas a una serie de ensayos para detectar su capacidad para producir biosurfactantes, así como la propiedad emulsificante y la estabilidad de la emulsión. 
Métodos de detección de la capacidad para producir biosurfactantes

Se determinó la capacidad para producir biosurfactante por medio de tres métodos. Todos los ensayos se realizaron al menos por triplicado.

\section{Actividad hemolitica}

Cada cepa se inoculó en una placa de agar sangre (\# de catálogo 220150, BBL®) y se incubó a $37^{\circ} \mathrm{C}$ durante $48 \mathrm{~h}$ como lo describe Youssef et al. (2004). Las placas fueron revisadas visualmente para la detección de halos de hemólisis alrededor de las colonias. Los biosurfactantes pueden causar la lisis de los eritrocitos de la sangre. La presencia de un halo de hemólisis alrededor de la cepa indica la lisis de los eritrocitos y por lo tanto, la presencia del biosurfactante (Walter et al. 2010).

\section{Gota colapsada (Drop collapse)}

Cada cepa se inoculó en un matraz Erlenmeyer que contenía $50 \mathrm{~mL}$ de caldo soya tripticaseína (TSB, \# de catálogo 211825 , Bacto $\left.{ }^{\circledR}\right)$ y se incubó a $37^{\circ} \mathrm{C}$ y a $150 \mathrm{rpm}$ durante $48 \mathrm{~h}$. Para separar el sobrenadante, los cultivos bacterianos fueron centrifugados a 6000 rpm durante $15 \mathrm{~min}$. Este ensayo se evaluó utilizando tres variantes, con la finalidad de conocer cuál de ellas se adecúa mejor a condiciones con alta salinidad.

\section{Variante 1 (Tugrul y Cansunar 2005)}

Se colocaron $7 \mu \mathrm{L}$ de aceite mineral en pozos de una placa de microtitulación de 96 pozos. La placa cubierta se dejó reposar por $24 \mathrm{~h}$ a temperatura ambiente. Posteriormente, a cada pozo se le adicionó una alícuota de $20 \mu \mathrm{L}$ del sobrenadante del cultivo bacteriano a ensayar. Después de 1 min la gota fue examinada visualmente. Como control positivo se utilizó una solución saturada de dodecil sulfato de sodio en medio de soya tripticaseína. Como testigo negativo se añadió agua destilada en lugar del surfactante al mismo medio.

\section{Variante 2 (Batista et al. 2006)}

Este ensayo se realizó con las mismas condiciones que la variante 1 pero utilizando aceite de oliva en lugar de aceite mineral.

\section{Variante 3 (Panjiar et al. 2015)}

A un tubo de ensayo que contenía $5 \mu \mathrm{L}$ de azul de metileno se le agregó una alícuota de $20 \mu \mathrm{L}$ del sobrenadante del cultivo bacteriano a ensayar. Una gota de esta mezcla se colocó sobre papel parafilm. La forma de la gota fue observada después de $10 \mathrm{~s}$. Como testigo positivo se utilizó una solución saturada de dodecil sulfato de sodio en medio de soya tripticaseína. Como testigo negativo se añadió agua destilada en lugar del surfactante al mismo medio.

Para las tres variantes de análisis, si el sobrenadante del cultivo bacteriano contiene biosurfactantes, las gotas se colapsan debido a una reducción de la tensión interfacial entre la gota del líquido y la superficie hidrófoba (Jain et al. 1991). En este caso las superficies hidrófobas fueron aceite mineral, aceite de oliva y papel parafilm, respectivamente.

\section{Difusión del diámetro de la gota de aceite (oil spreading)}

Este método consiste en añadir $40 \mathrm{~mL}$ de agua destilada a una caja de Petri vacía, adicionándole 50 $\mu \mathrm{L}$ de aceite vegetal y $10 \mu \mathrm{L}$ del sobrenadante del cultivo bacteriano en un medio de soya tripticaseína. Se midió el diámetro de la zona clara alrededor de la suspensión formada, según describe Sharma et al. (2014). El cambio de la tensión superficial producido por el biosurfactante es capaz de desplazar el aceite. El diámetro de la zona clara en la superficie del aceite se correlaciona con la capacidad para producir biosurfactante (Morikawa et al. 1993).

Método de detección de la propiedad emulsificante Índice de emulsificación (\% I.E.) (Plaza et al. 2006)

Se determinó adicionando $2 \mathrm{~mL}$ del sobrenadante del cultivo bacteriano en medio soya tripticaseína a $2 \mathrm{~mL}$ de tolueno en un tubo de ensayo. Esta mezcla se agitó con vórtex a alta velocidad durante $2 \mathrm{~min}$ y luego se dejó reposar durante $24 \mathrm{~h}$. El índice de emulsificación $\left(\mathrm{E}_{24}\right)$ se define como la relación entre la altura de la capa de emulsión dividida entre la altura total y expresada como porcentaje.

Estabilidad de la emulsión (E.E.) (Das et al. 1998)

Este parámetro se determinó a partir del volumen de emulsión (VE) relativa medido en el intervalo de 0 a $48 \mathrm{~h}$, cada $24 \mathrm{~h}$ con las siguientes ecuaciones:

$$
\begin{aligned}
& \text { altura de la emulsión ( } \mathrm{mm} \text { ) } x \text { área } \\
& V E=\frac{\text { de sección transversal }\left(\mathrm{mm}^{2}\right)}{\text { volumen total del líquido }\left(\mathrm{mm}^{3}\right)} \times 100 \\
& E E=\frac{\text { VE al tiempo } t,(h)}{\text { VE al tiempo } 0(h)} \times 100
\end{aligned}
$$

Un criterio citado para definir la estabilidad de la emulsión es que el parámetro E.E. sea mayor a $50 \%$ después de $48 \mathrm{~h}$. Las estabilidades de las emulsiones formadas fueron comparadas con una solución $1 \%$ de dodecil sulfato de sodio en agua desionizada. 


\section{Caracterización fisiológica}

Se determinó la concentración de $\mathrm{NaCl}$ y el valor de $\mathrm{pH}$ para el crecimiento óptimo de las cepas aisladas mediante la inoculación de cada cepa en el medio $\mathrm{MH}$ complementado con $\mathrm{NaCl}$ a las siguientes concentraciones: $0,0.5,3,5,10,15,20,25$ y $30 \%$. $\mathrm{El} \mathrm{pH}$ fue ajustado en cada concentración de 5 a 12. El inóculo se sembró y se mantuvo en incubación a $37^{\circ} \mathrm{C}$. El crecimiento se observó diariamente por $10 \mathrm{~d}$.

\section{Identificación genética}

A las cepas aisladas del ex lago de Texcoco las cuales demostraron capacidad para producir biosurfactantes por al menos uno de los métodos ensayados, se les realizó una identificación genética por análisis de secuenciación del gen 16S ARNr. La extracción de ADN se hizo de acuerdo con el protocolo del paquete Promega Wizard ${ }^{\circledR}$ purificación de ADN genómico (Promega, A1120). Se realizó la amplificación por reacción en cadena de la polimerasa (PCR, por sus siglas en inglés) del gen 16S ARNr, los iniciadores utilizados fueron los siguientes: $8 \mathrm{~F}$ : AGAGTTTGATCMTGGCTCAG y 1492r: TACGGYTACCTTGTTACGACTT. La reacción se llevó a cabo con la enzima Taq ADN polimerasa comercial (Dongsheng Biotech, P1082). Los fragmentos amplificados del gen 16S ARNr fueron filtrados a través de un equipo de purificación PCR Amicon Ultra 0.5 $\mathrm{mL}$ (Merck Millipore, UFC503096) de acuerdo con la metodología proporcionada por el fabricante. Los productos amplificados fueron enviados a un servicio de secuenciación (Macrogen, Maryland, EUA) y las secuencias obtenidas se revisaron y corrigieron. Las secuencias consenso se construyeron con el programa Bioedit versión 7.0.9 (Hall 1999). Para determinar el porcentaje de semejanza de las secuencias consenso, se compararon con secuencias ya depositadas en la base de datos "GenBank" (Centro Nacional de Información sobre Biotecnología - NCBI, por sus siglas en inglés) con el programa de herramienta de búsqueda para alineamiento local básico (BLAST, por sus siglas en inglés; Altschul 1990).

\section{Utilización de antraceno como única fuente de carbono y energía}

Las cepas que mostraron capacidad para producir biosurfactantes con propiedad emulsificante y estabilidad en la emulsión fueron sembradas en el medio mínimo de sales (MSM, por sus siglas en inglés) adicionado con antraceno, para determinar su capacidad de utilizarlo como única fuente de carbono y energía.

\section{Medio de cultivo}

Se utilizó MSM (Sang et al. 2009): $\left(\mathrm{NH}_{4}\right)_{2} \mathrm{SO}_{4}$, $2000 \mathrm{mg} / \mathrm{L} ; \mathrm{Na}_{2} \mathrm{HPO}_{4}, 1600 \mathrm{mg} / \mathrm{L} ; \mathrm{K}_{2} \mathrm{HPO}_{4}$, $400 \mathrm{mg} / \mathrm{L} ; \mathrm{MgSO}_{4} \cdot 7 \mathrm{H}_{2} \mathrm{O}, 400 \mathrm{mg} / \mathrm{L} ; \mathrm{CaCl}_{2} \cdot 2 \mathrm{H}_{2} \mathrm{O}$, $200 \mathrm{mg} / \mathrm{L} ; \mathrm{FeCl}_{3} \cdot \mathrm{H}_{2} \mathrm{O}, 10 \mathrm{mg} / \mathrm{L} ;\left(\mathrm{NH}_{4}\right)_{6} \mathrm{Mo}_{7} \mathrm{O}_{24} \cdot \mathrm{H}_{2} \mathrm{O}$, $2 \mathrm{mg} / \mathrm{L}$. A este medio se le adicionó la concentración de $\mathrm{NaCl}$ óptima para el crecimiento de cada cepa y se ajustó el $\mathrm{pH}$ con el mismo fin. Cada medio fue esterilizado en autoclave a $121^{\circ} \mathrm{C}$ durante $15 \mathrm{~min}$.

El medio se dejó enfriar a temperatura ambiente y se le adicionó $3.3 \mathrm{~mL}$ de una solución de antraceno previamente preparada en medio MSM $(0.3 \mu \mathrm{g} / \mathrm{mL})$ y se esterilizó por filtración (Millipore filter, Millex with Durapore membrane of $0.22 \mu \mathrm{m}$, SLGC033RS). A este medio que contiene antraceno se le llamó MSM-Ant, el cual se cubrió con papel de aluminio.

\section{Cinética de crecimiento en MSM con antraceno}

Para caracterizar la curva de crecimiento de cada cepa, se preparó el preinóculo transfiriendo una colonia del cultivo bacteriano a $60 \mathrm{~mL}$ del medio de cultivo MSM-Ant contenido en matraces con tapón de rosca cubierto con papel aluminio. Se incubaron a $37^{\circ} \mathrm{C}$ y a $150 \mathrm{rpm}$. El crecimiento microbiano se determinó al medir su densidad óptica a $600 \mathrm{~nm}$ (Nnamchi et al. 2006) en un espectrofotómetro (Perkin-Elmer UV-Vis, modelo 551S) a diferentes tiempos hasta alcanzar la fase estacionaria.

Una vez conocida la duración de la fase exponencial para cada cepa, se tomó un volumen de $250 \mu \mathrm{L}$ a la mitad de esta fase, el cual fue ajustado a una $\mathrm{DO}_{600 \mathrm{~nm}}=0.25$. A partir de este volumen ajustado se tomó un inóculo de $1 \mathrm{~mL}$. Este $\mathrm{mL}$ fue transferido a un matraz con tapón de rosca cubierto con papel aluminio que contenía $60 \mathrm{~mL}$ de MSM-Ant. Los matraces fueron incubados a $150 \mathrm{rpm}$ y $37^{\circ} \mathrm{C}$. El crecimiento microbiano se determinó midiendo su densidad óptica a $600 \mathrm{~nm}$ (Nnamchi et al. 2006) en un espectrofotómetro (Perkin-Elmer UV-Vis, modelo 551S) a diferentes tiempos hasta alcanzar la fase estacionaria.

\section{RESULTADOS}

\section{Cepas de actinobacterias con capacidad para producir biosurfactantes}

De las muestras de suelo obtenidas del ex lago de Texcoco se aislaron un total de 11 cepas de actinobacterias, las cuales presentaron características morfológicas como colonias secas, rugosas, con presencia de micelio aéreo. Todas las cepas fueron Gram-positivas filamentosas y presentaron micelio ramificado. Sus 
claves de identificación inician con las letras TX... Estas cepas, más las siete provenientes de suelos de Veracruz, las cuales tienen como clave M..., hacen un total de 18 cepas. De estas 18 cepas, 11 mostraron capacidad para producir biosurfactantes por al menos uno de los ensayos. De estas 11, siete son las cepas anteriormente aisladas del estado de Veracruz que tienen la propiedad de transformar antraceno: $\mathrm{M} 2 \mathrm{C}, \mathrm{M} 3 \mathrm{H}$, M3I, M5B, M11A, M10A, M1B (Lara-Severino et al. 2016a). Las cuatro restantes provienen del ex lago de Texcoco: TX5SEC-8SG2, TX5SEC-4MHG6, TXV11-13SG3, TX5SEC-3MHG6. Los resultados de estas 11 cepas con capacidad para producir biosurfactantes están resumidos en el cuadro I. Dicho cuadro I muestra además que, cinco de las cepas estudiadas tienen capacidad para producir biosurfactantes con propiedades emulsificantes. El porcentaje de la E.E. determinado a las $48 \mathrm{~h}$ se encuentra en el intervalo de 69 a 97 \%. Esto indica que la emulsión formada es estable al menos por ese periodo de tiempo según el criterio de Das et al. (1998).

\section{Caracterización fisiológica e identificación gené- tica}

Las cepas fueron caracterizadas fisiológicamente de acuerdo con el porcentaje de $\mathrm{NaCl}$ y el valor de $\mathrm{pH}$ en donde mostraron un crecimiento óptimo. Las cepas aisladas de los suelos de Veracruz todas fueron caracterizadas como haloalcalotolerantes y las aisladas del ex lago de Texcoco como haloalcalófilas. Estas últimas fueron identificadas como Nocardiopsis salina (3 cepas) y Nocardiopsis exhalans (1 cepa). El cuadro II muestra los resultados de la caracterización fisiológica de las 11 cepas con capacidad para producir biosurfactantes, así como el número de pares de bases del fragmento amplificado en la identificación genética y el porcentaje de semejanza con la especie correspondiente.

\section{Utilización de antraceno como única fuente de carbono y energía}

Las cinco cepas que demostraron capacidad para producir biosurfactantes con propiedades emulsificantes y estabilidad en la emulsión (M11A, M10A, TXV11-13SG3, TX5SEC-3MHG6, M1B), también mostraron capacidad para utilizar al antraceno como única fuente de carbono y energía. En la figura $1 \mathrm{se}$ muestra la curva de crecimiento con una fase exponencial acelerada durante la primera hora, la cual continúa de forma moderada durante las siguientes 5 $\mathrm{h}$, a partir de la cual se alcanza la fase estacionaria. La figura 1 muestra que estas cepas utilizan antraceno como única fuente de carbono y energía, pero difieren ligeramente en la velocidad de crecimiento bajo las condiciones de este estudio.

\section{DISCUSIÓN}

En los últimos años se ha incrementado la búsqueda de microrganismos con capacidad biodegradadora como una alternativa para mitigar los problemas de contaminación ambiental por HPA y derivados del petróleo. Dada la baja solubilidad de estos compuestos en soluciones acuosas, una característica deseable en estos microorganismos es su capacidad para producir biosurfactantes con propiedades emulsificantes con la finalidad de aumentar la solubilidad del HPA o derivado del petróleo contaminante y su biodisponibilidad. Dentro de los microrganismos que cumplen con esta característica se encuentran las actinobacterias.

Por otro lado, varios ambientes contaminados con hidrocarburos se caracterizan por presentar altas concentraciones de sal (Margesin y Schinner 2001). Las actinobacterias haloalcalófilas y haloalcalotolerantes están adaptadas a crecer en estas condiciones salinas. Por estas razones en este trabajo se estudió la capacidad para producir biosurfactantes con propiedades emulsificantes por métodos sencillos, rápidos, baratos y convencionales a 18 actinobacterias provenientes de suelos salinos (ex lago de Texcoco) o ligeramente salinos (Veracruz).

El método de la gota colapsada y el de la difusión del diámetro de la gota de aceite se consideran los más sensibles para detectar la capacidad de producir biosurfactante (Youssef et al. 2004, Satpute et al. 2008). Cabe mencionar que no todos los biosurfactantes tienen actividad hemolítica (Youssef et al. 2004), por lo que la negatividad de este ensayo tiene dos variantes: a) o no hay actividad biosurfactante $o$ b) hay actividad biosurfactante pero no actividad hemolítica. Por lo tanto, esta prueba debe utilizarse siempre como complemento de los métodos de la gota colapsada y el de la difusión del diámetro de la gota de aceite, cuando se evalúa la capacidad de un microorganismo para producir biosurfactante.

Los resultados correspondientes a las cepas que no mostraron capacidad para producir biosurfactantes por el método de actividad hemolítica ni propiedad emulsificante (cuadro I) no son concluyentes, ya que hay dos posibilidades: a) las cepas tienen capacidad para producir biosurfactante (demostrada por los otros métodos), pero sin propiedades emulsificantes o b) las condiciones de los ensayos no fueron las que garantizan la mayor sensibilidad $(\mathrm{pH}, \% \mathrm{NaCl}$, tiempo de incubación, etcétera). 

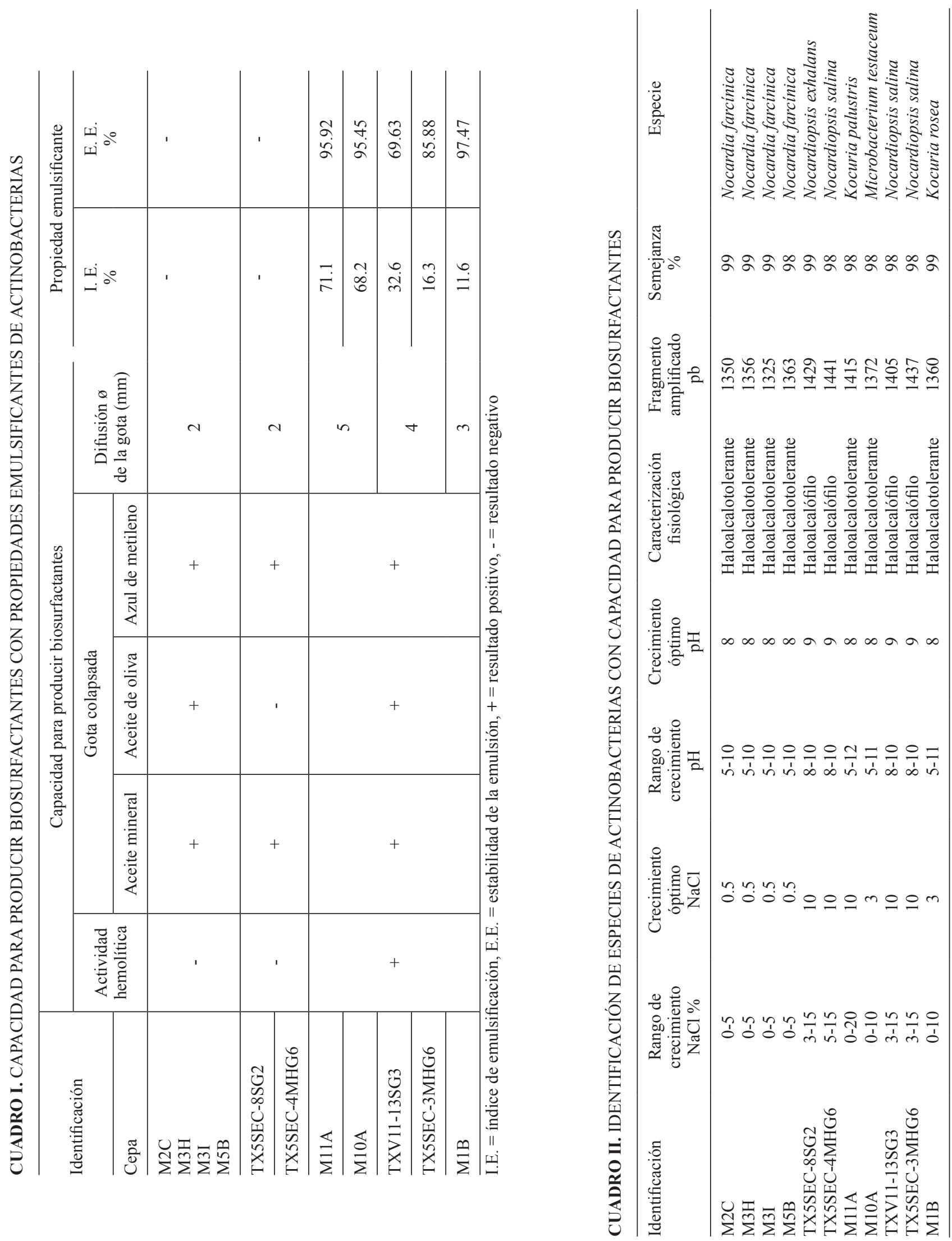


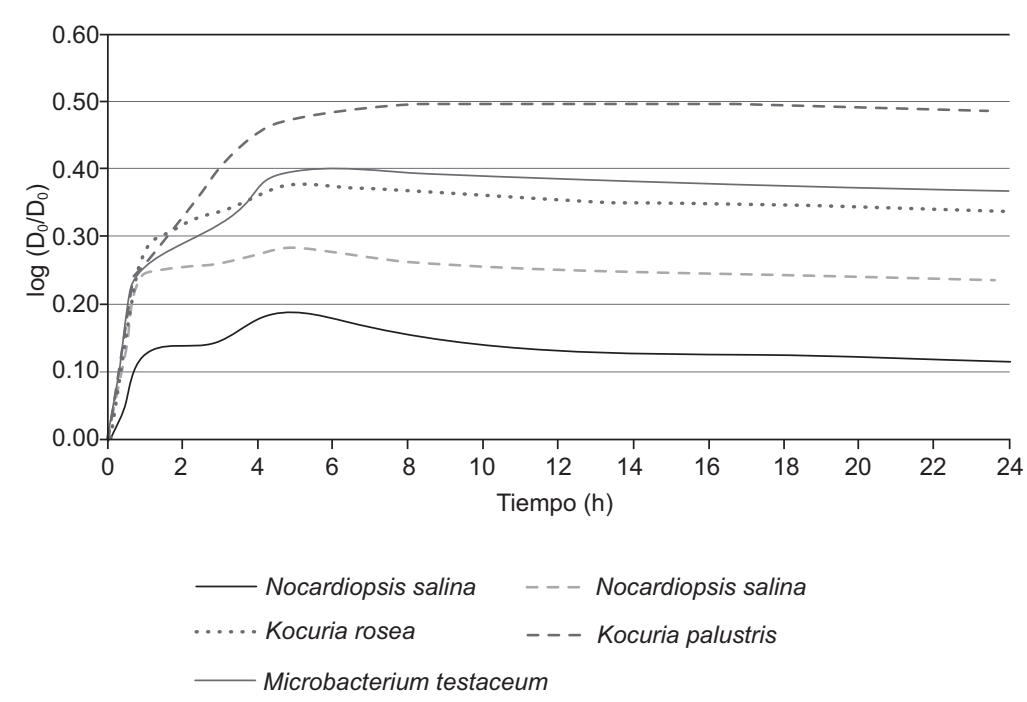

Fig. 1. Cinéticas de crecimiento en medio de sales mínimo con antraceno de dos cepas haloalcalófilas (Nocardiopsis salina) y de tres cepas haloalcalotolerantes (Kocuria palustris, Microbacterium testaceum y Kocuria rosea). El crecimiento está expresado en función de la densidad óptica (DO), donde $\mathrm{DO}_{0}$ es la densidad óptica inicial (al tiempo $\mathrm{t}=0$ )

En este trabajo las cepas de las actinobacterias Kocuria palustris (una cepa haloalcalotolerante), Microbacterium testaceum (una cepa haloalcalotolerante), Nocardiopsis salina (dos cepas haloalcalófilas) y Kocuria rosea (una cepa haloalcalotolerante) fueron positivas a todas las variantes del ensayo de la gota colapsada, además también resultaron positivas al de difusión del diámetro de la gota y tuvieron actividad hemolítica (cuadro I). Asimismo, estas cepas mostraron propiedades emulsificantes, cuyas emulsiones formadas fueron estables. La figura 1 muestra además que utilizan antraceno como única fuente de carbono y energía, ya que son capaces de crecer en presencia de éste. Cabe destacar que en el caso de la Kocuria palustris, Kocuria rosea y Microbacterium testaceum es conocido que transforman al antraceno (Lara-Severino et al. 2016a, 2016b). Por lo tanto, podemos plantear que: (i) dada la capacidad productora de biosurfactates con características emulsificantes y su capacidad para utilizar antraceno como única fuente de carbono y energía, las dos cepas de Nocardiopsis salina deberían seguir bajo estudio a fin de determinar si son capaces de transformar HPA como lo es el antraceno, (ii) Kocuria palustris, Kocuria rosea y Microbacterium testaceum tienen capacidad productora de biosurfactantes, son capaces de utilizar antraceno como única fuente de carbono y energía y transformarlo, por lo que son candidatas potenciales para ser utilizadas en procesos de biorremediación de ambientes salinos contaminados con hidrocarburos policíclicos aromáticos.
Kocuria palustris, según Mariano et al. (2008) degrada diésel. En el presente artículo esta especie creció en presencia de antraceno. Sin embargo, no hay reportes de la capacidad de este microorganismo en producir biosurfactante con propiedades emulsificantes. En este estudio esta cepa resultó tener el mayor índice de emulsificación y una muy alta estabilidad de la emulsión.

La cepa Microbacterium testaceum mostró capacidad para producir biosurfactante con propiedades emulsificantes, así como capacidad para crecer en presencia de antraceno. Sin embargo, hasta el momento no hay reportes que indiquen este comportamiento. Aniszewski et al. (2010) reportaron la capacidad biosurfactante con propiedad emulsificante de Microbacterium sp., mientras que Vasconcellos et al. (2009) reportan su capacidad degradadora de dihidrofenantreno.

De manera similar ocurre con la especie Nocardiopsis salina, en este trabajo se reporta la capacidad de dos cepas en producir biosurfactante con propiedades emulsificantes, además de crecer en presencia de antraceno y no existen reportes similares. Otros autores demostraron la capacidad de producir biosurfactante y bioemulsificante en otras especies de este género como Nocardiopsis alba (Gandhimathi et al. 2009) y Nocardiopsis lucentensis (Kiran et al. 2010). Por otro lado, Dixit y Pant (2000) reportaron la capacidad degradadora de petróleo de Nocardiopsis sp.

La capacidad para producir biosurfactantes con propiedades emulsificantes y la capacidad 
biodegradadora de antraceno de Kocuria rosea ha sido anteriormente reportada (Ahmed et al. 2010, Mahjoubi et al. 2013), lo que concuerda con nuestros resultados.

\section{CONCLUSIONES}

De 18 actinobacterias evaluadas, cinco cepas mostraron capacidad para producir biosurfactantes con propiedades emulsificantes, alta estabilidad en la emulsión y utilización de antraceno como única fuente de carbono y energía. De estas cinco cepas, dos son haloalcalófilas y pertenecen a la especie Nocardiopsis salina, mientras que tres son haloalcalotolerantes y pertenecen a las especies Kocuria palustris, Microbacterium testaceum y Kocuria rosea. Los métodos de la gota colapsada, difusión del diámetro de la gota de aceite y actividad hemolítica son métodos complementarios, sencillos, rápidos y fáciles para detectar capacidad productora de biosurfactante en el sobrenadante de cultivos bacterianos de actinobacterias haloalcalófilas y haloalcalotolerantes. La capacidad de Nocardiopsis salina para trasformar HPA no fue investigada en este trabajo y la capacidad de Kocuria palustris, Kocuria rosea y Microbacterium testaceum para transformar antraceno ya se conocía con anterioridad. Dado que las características ideales de un microrganismo para considerarse potencialmente degradador son la capacidad de producir biosurfactantes con características emulsificantes y la capacidad de transformar HPA (como el antraceno), se concluye que Kocuria palustris, Kocuria rosea y Microbacterium testaceum son potenciales candidatas para ser utilizadas en procesos de biorremediación de ambientes salinos contaminados con HPA.

\section{AGRADECIMIENTOS}

Los autores agradecen la asistencia financiera de la Secretaría de Investigación y Estudios Avanzados de la Universidad Autónoma del Estado de México (UAEMex) a través del proyecto de investigación, "Estudio bioquímico y molecular de actinomicetos presentes en suelos contaminados con hidrocarburos" 3690/2014/CID, así como el apoyo mediante la Red Académica y de colaboración: "Microbiología y Química en Ciencias de la Salud 1039/2014RIFC". Los autores agradecen al Programa para el Desarrollo Profesional Docente (PRODEP) de la Secretaria de Educación Pública (SEP), en especial a su Convocatoria de Redes 2015, por el apoyo financiero brindado a la "Red-hispano-mexicana para la búsqueda y aprovechamiento de microorganismos extremófilos con aplicaciones ambientales y biomédicas", la cual actualmente ejecuta el proyecto "Estudio de la degradación de hidrocarburos policíclicos aromáticos por actinomicetos halo-alcalotolerantes utilizando métodos de espectrofluorimetría". Este trabajo se deriva de la tesis doctoral de Reyna del Carmen Lara Severino estudiante del programa de Doctorado en Ciencias de la Salud de la Universidad Autónoma del Estado de México inscrito en el Programa Nacional de Posgrados de Calidad del Consejo Nacional de Ciencia y Tecnología (PNPC-CONACYT).

\section{CONFLICTO DE INTERESES}

Los autores declaran no tener ningún conflicto de intereses financieros, académicos, comerciales, políticos o personales.

\section{REFERENCIAS}

Ahmed R. Z., Ahmed N. y Gadd G. M. (2010). Isolation of two Kocuria species capable of growing on various polycyclic aromatic hydrocarbons. Afr. J. Biotechnol. 9 (24), 3611-3617. DOI: 10.5897/AJB2010.000-3224

Al-Awadhi H., Al-Mailem D., Dashti N., Khanafer M. y Radwan S. (2012). Indigenous hydrocarbon-utilizing bacterioflora in oil-polluted habitats in Kuwait, two decades after the greatest man-made oil spill. Arch. Microbiol. 194 (8), 689-705.

DOI: $10.1007 / \mathrm{s} 00203-012-0800-7$

Altschul S. F., Gish W., Miller W., Myers E. W. y Lipman D. J. (1990). Basic local alignment search tool. J. Mol. Biol. 215 (3), 403-410. DOI: 10.1016/S0022-2836(05)80360-2

Aniszewski E., Peixoto R. S., Mota F. F., Leite S. G. F. y Rosado A. S. (2010). Bioemulsifier production by Microbacterium sp. strains isolated from mangrove and their application to remove cadmiun and zinc from hazardous industrial residue. Braz. J. Microbiol. 41 (1), 235-245.

DOI: $10.1590 / \mathrm{S} 1517-838220100001000033$

Batista S. B., Mounteer A. H., Amorim F. R. y Tótola M. R. (2006). Isolation and characterization of biosurfactant/ bioemulsifier-producing bacteria from petroleum contaminated sites. Bioresource technol. 97 (6), 868-875. DOI: 10.1016/j.biortech.2005.04.020

Bognolo G. (1999). Biosurfactants as emulsifying agents for hydrocarbons. Colloids Surf., A 152 (1-2), 41-52. DOI: $10.1016 / \mathrm{S} 0927-7757(98) 00684-0$ 
Bonnet J. L., Guiraud P., Dusser M., Kadri M., Laffosse J., Steiman R. y Bohatier J. (2005). Assessment of anthracene toxicity toward environmental eukaryotic microorganisms: Tetrahymena pyriformis and selected micromycetes. Ecotox. Environ. Safe 60 (1), 87-100. DOI: 10.1016/j.ecoenv.2003.10.001

Das M., Das S. K. y Mukherjee R. K. (1998). Surface active properties of the culture filtrates of a Micrococcus species grown on n-alkanes and sugars. Bioresource Technol. 63 (3), 231-235.

DOI: $10.1016 / \mathrm{S} 0960-8524(97) 00133-8$

de Vasconcellos S. P., Crespim E., da Cruz G. F., Senatore D. B., Simioni K. C. M., Neto E. V. D. S., Marsaioli A. J. y de Oliveira V. M. (2009). Isolation, biodegradation ability and molecular detection of hydrocarbon degrading bacteria in petroleum samples from a Brazilian offshore basin. Org. Geochem. 40 (5), 574-588.

DOI: 10.1016/j.orggeochem.2009.02.006

Dixit V. S. y Pant A. (2000). Hydrocarbon degradation and protease production by Nocardiopsis sp. NCIM 5124. Lett. Appl. Microbiol. 30 (1), 67-69.

DOI: $10.1046 / j .1472-765 x .2000 .00665 . x$

Ferradji F. Z., Mnif S., Badis A., Rebbani S., Fodil D., Eddouaouda K. y Sayadi S. (2014). Naphthalene and crude oil degradation by biosurfactant producing Streptomyces spp. isolated from Mitidja plain soil (North of Algeria). Int. Biodeter. Biodegr. 86 (Part C), 300-308. DOI: 10.1016/j.ibiod.2013.10.003

Gandhimathi R., Kiran G. S., Hema T. A., Selvin J., Raviji T. R. y Shanmughapriya S. (2009). Production and characterization of lipopeptide biosurfactant by a sponge-associated marine actinomycetes Nocardiopsis alba MSA10. Bioprocess Biosyst. Eng. 32 (6), 825835. DOI: $10.1007 / \mathrm{s} 00449-009-0309-x$

Hall T. A. (1999). BioEdit: a user-friendly biological sequence alignment editor and analysis program for Windows 95/98/NT. Nucl. Acids Symp. Ser. 41, 95-98.

Holt J. G., Krieg N. R., Sneath P. H. A., Staley J. T. y Williams S. T.(1994). Bergey's manual of determinative bacteriology. 9a ed. Lippicontt Williams and Wilkins. Maryland, EUA, 787 pp.

Horikoshi K. (1999). Alkaliphiles: Some applications of their products for biotechnology. Microbiol. Mol. Biol. Rev. 63 (4), 735-750.

Jain D. K., Collins-Thompson D. L., Lee H. y Trevors J. T. (1991). A drop-collapsing test for screening surfactantproducing microorganisms. J. Microbiol. Methods 13 (4), 271-279.

DOI: 10.1016/0167-7012(91)90064-W

Jain P. K., Gupta V. K., Gaur R. K., Lowry M., Jaroli D. P. y Chauhan U. K. (2011). Bioremediation of petroleum oil contaminated soil and water. Res. J. Environl. Toxic. 5 (1), 1-26. DOI: 10.3923/rjet.2011.1.26
Juhasz A. L. y Naidu R. (2000). Bioremediation of high molecular weight polycyclic aromatic hydrocarbons: a review of the microbial degradation of benzo(a)pyrene. Int. Biodeter. Biodegr. 45 (1-2), 57-88.

DOI: $10.1016 / \mathrm{S} 0964-8305(00) 00052-4$

Kiran G. S., Thomas T. A. y Selvin J. (2010). Production of a new glycolipid biosurfactant from marine Nocardiopsis lucentensis MSA04 in solid-state cultivation. Colloids Surf. B 78 (1), 8-16.

DOI: $10.1016 /$ j.colsurfb.2010.01.028

Kügler J. H., Roes-Hill M. L., Syldatk C. y Hausmann R. (2015). Surfactants tailored by the class Actinobacteria. Front. Microbiol. 6, 212, 1-23.

DOI: $10.3389 /$ fmicb.2015.00212

Kushner D. J. y Kamekura M. (1988). Physiology of halophilic eubacteria. En: Halophilic bacteria. (F. Rodríguez-Valera, Ed.). CRC Press, Boca Raton, Florida, EUA, pp. 109-138.

Lai Ch. Ch., Huang Y. Ch., Wei Y. H. y Chang J. S. (2009). Biosurfactant-enhanced removal of total petroleum hydrocarbons from contaminated soil. J. Hazard. Mater. 167 (1-3), 609-614.

DOI: $10.1016 /$ j.jhazmat.2009.01.017

Lara-Severino R. C., Camacho-López M. A., CasanovaGonzález E, Gómez-Oliván L. M., Sandoval-Trujillo A. H., Isaac-Olivé K. y Ramírez-Durán N. (2016a). Haloalkalitolerant actinomycetes with capacity for anthracene degradation isolated from soils close to areas with oil activity. Int. Microbiol. 19 (1), 15-26. DOI: 10.2436/20.1501.01.259

Lara-Severino R. C., Camacho-López M. A., GarcíaMacedo J. M., Gómez-Oliván L. M., Sandoval-Trujillo A. H., Isaac-Olivé K. y Ramírez-Durán N. (2016b). Determination of the residual anthracene concentration in cultures of haloalkalitolerant actinomycetes by excitation fluorescence, emission fluorescence, and synchronous fluorescence: comparative study. J. Anal. Methods Chem. Article ID 6287931.

DOI: $10.1155 / 2016 / 6287931$

Mahjoubi M., Jaouani A., Guesmi A., Amor S. B., Jouini A., Cherif H., Najjari A., Boudabous A., Koubaa N. y Cherif A. (2013). Hydrocarbonoclastic bacteria isolated from petroleum contaminated sites in Tunisia: isolation, identification and characterization of the biotechnological potential. N. Biotechnol. 30 (6), 723-733.

DOI: $10.1016 /$ j.nbt.2013.03.004

Margesin R. y Schinner F. (2001). Biodegradation and bioremediation of hydrocarbons in extreme environments. Appl. Microbiol. Biotechnol. 56 (5-6), 650-663. DOI: $10.1007 / \mathrm{s} 002530100701$

Mariano A. P., Bonotto D. M., de Angelis D. D. F., Piróllo M. P. S. y Contiero J. (2008). Biodegradability of 
commercial and weathered diesel oils. Braz. J. Microbiol. 39 (1):133-142.

DOI: $10.1590 / \mathrm{S} 1517-83822008000100028$

Morikawa M., Daido H., Takao T. Murata S. Shimonishi Y. e Imanaka T. (1993). A new lipopeptide biosurfactant produced by Arthrobacter sp. strain MIS38. J. Bacteriol. 175 (20), 6459-6466.

Mukherjee S., Das P. y Sen R. (2006). Towards commercial production of microbial surfactants. Trends Biotechnol. 24 (11), 509-515. DOI: 10.1016/j.tibtech.2006.09.005

Mulligan C. N. (2005). Environmental applications for biosurfactants. Environ. Pollut. 133 (2), 183-198. DOI: 10.1016/j.envpol.2004.06.009

Nnamchi C. I., Obeta J. A. N. y Ezeogu L. I. (2006). Isolation and characterization of some polycyclic aromatic hydrocarbon degrading bacteria from Nsukka soils in Nigeria. Int. J. Environ. Sci. Tech. 3 (2), 181-190. DOI: $10.1007 / \mathrm{BF} 03325924$

Oren A. (2005). Halophilic microorganisms: Physiology and phylogeny. En: Origins (J. Seckbach, Ed.). Springer, Holanda, pp. 413-426.

Panjiar N., Sachan S. G. y Sachan A. (2015). Screening of bioemulsifier-producing micro-organisms isolated from oil-contaminated sites. Ann. Microbiol. 65 (2), 753-764. DOI: 10.1007/s13213-014-0915-y

Plaza G. A., Zjawiony I. y Banat I. M. (2006). Use of different methods for detection of thermophilic biosurfactantproducing bacteria from hydrocarboncontaminated and bioremediated soils. J. Petrol. Sci. Eng. 50 (1), 71-77.

DOI: 10.1016/j.petrol.2005.10.005

Quesada E., Ventosa A., Rodriguez-Valera F., Megias L. y Ramos-Cormenzana A. (1983). Numerical taxonomy of moderately halophilic Gram-negative bacteria from hypersaline soils. J. Gen. Microbiol. 129, 2649-2657. DOI: 10.1099/00221287-129-8-2649

Redman A. D., McGrath J. A., Stubblefield W. A., Maki A. W. y Di Toro D. M. (2012). Quantifying the concentration of crude oil microdroplets in oil-water preparations. Environ. Toxicol. Chem. 31 (8), 1814-1822. DOI: $10.1002 /$ etc. 1882

Sang L. Z., Wei X. Y., Chen J. N., Zhu Y. X. y Zhang Y. (2009) Simultaneous fluorimetric determination of the biodegradation processes of dissolved multicomponent PAHs. Talanta 78 (4-5), 1339-1344. DOI: 10.1016/j.talanta.2009.02.009

Sarafin Y., Donio M. B. S., Velmurugan S., Michaelbabu M. y Citarasu T. (2014). Kocuria marina BS-15 a biosurfactant producing halophilic bacteria isolated from solar salt works in India. Saudi J. Biol. Sci. 21 (6), 511-519. DOI: 10.1016/j.sjbs.2014.01.001
Satpute S. K., Bhawsar B. D., Dhakephalkar P. K. y Chopade B. A. (2008). Assessment of different screening methods for selecting biosurfactant producing marine bacteria. Indian J. Mar. Sci. 37 (3), 243-250.

Sharma A., Soni J., Kaur G. y Kaur J. (2014). A study on biosurfactant production in Lactobacillus and Bacillus sp. Int. J. Curr. Microbiol. App. Sci. 3 (11), 723-733.

Soberón-Chávez G. y Maier R. M. (2011). Biosurfactants: a general overview. En: Biosurfactants (G. SoberónChávez, Ed.). Springer, Berlin, Alemania, pp 1-11.

Tugrul T. y Cansunar E. (2005). Detecting surfactantproducing microorganisms by the drop-collapse test. World J. Microb. Biot. 21, 851-853.

DOI: $10.1007 / \mathrm{s} 11274-004-5958-\mathrm{y}$

USEPA (1990). Anthracene; CASRN 120-12-7. Integrated Risk Information System (IRIS). Chemical Assessment Summary. United States Environmental Protection Agency. [en línea]. https://cfpub.epa.gov/ncea/ iris $2 /$ chemicalLanding.cfm? substance_nmbr $=434$ 25/09/2016

Vyas T. K. y Dave B. P. (2011). Production of biosurfactant by Nocardia otitidiscaviarum and its role in biodegradation of crude oil. Int. J. Environ. Sci. Tech. 8 (2), 425-432. DOI: 10.1007/BF03326229

Walter V., Syldatk C. y Hausmann R. (2010). Screening concepts for the isolation of biosurfactant producing microorganisms. En: Biosurfactants (R. Sen, Ed.). Springer, Nueva York, EUA, pp. 1-13.

Yañez-Ocampo G. y Wong-Villarreal A. (2013). Biosurfactantes microbianos, producción potencial con residuos agroindustriales de Chiapas. Revista BioTecnología 17 (3), 12-28.

Youssef N. H., Duncan K. E., Nagle D. P., Savage K. N., Knapp R. M. y McInerney M. J. (2004). Comparison of methods to detect biosurfactant production by diverse microorganisms. J. Microbiol. Meth. 56 (3), 339-347. DOI: $10.1016 /$ j.mimet.2003.11.001

Zhong Y., Luan T., Wang X., Lan C. y Tam N. F. Y. (2007). Influence of growth medium on cometabolic degradation of polycyclic aromatic hydrocarbons by Sphingomonas sp. strain PheB4. Appl. Microbiol. Biotechnol. 75 (1), 175-186. DOI: $10.1007 / \mathrm{s} 00253-006-0789-4$

Zhu H. y Aitken M. D. (2010). Surfactant-enhanced desorption and biodegradation of polycyclic aromatic hydrocarbons in contaminated soil. Environ. Sci. Technol. 44 (19), 7260-7265. DOI: 10.1021/es100112a 